\title{
Polynômes singuliers à plusieurs variables sur un corps fini et congruences modulo $p^{2}$
}

par

\author{
El Mostafa Hanine (Toulouse)
}

1. Introduction. Dans ce travail on s'intéresse à la classification des polynômes singuliers de degré 4 sur un corps fini et aux congruences modulo $p^{2}$. Dans toute la suite $\mathbb{K}$ désignera un corps fini de caractéristique $p$ à $q=p^{a}$ éléments, $p$ un nombre premier impair et $\overline{\mathbb{K}}$ une clôture algébrique de $\mathbb{K}$.

Cette étude s'article comme suit :

- démontrer tout d'abord que si Card $\mathbb{K}>m^{2}$, tout $f \in \mathbb{K}[X]$ de degré $m$, à valeurs carrées dans $\mathbb{K}$, est un carré dans $\mathbb{K}[X]$,

- généraliser un théorème de Carlitz [2] au cas de polynômes à plusieurs variables,

- démontrer aussi que pour tout entier $n \geq 9$ et pour tout $F \in \mathbb{K}\left[X_{1}, \ldots\right.$ $\left.\ldots, X_{n}\right]$ singulier de degré 4 sans terme constant, si $q \geq 37$ il existe $v \in \mathbb{K}$ non carré et $g_{1}, g_{2}$ éléments de $\mathbb{K}\left[X_{1}, \ldots, X_{n}\right]$ de degré 2 sans termes constants tels que

$$
F=\varepsilon\left(g_{1}^{2}-v g_{2}^{2}\right) \quad \text { où } \varepsilon= \pm 1 .
$$

Ceci généralise le résultat de D. J. Lewis [5].

Dans [3] on a démontré que pour tout entier $d \geq 1$, il existe des nombres premiers dont la plus petite valeur, $p(d)$, vérifie la propriété suivante : pour tout $p$ nombre premier $\geq p(d)$ et pour tout $F \in \mathbb{Z}_{p}\left[X_{1}, \ldots, X_{2 d+1}\right]$ de degré $d$ sans terme constant, l'équation $F\left(x_{1}, \ldots, x_{2 d+1}\right) \equiv 0\left(\bmod p^{2}\right)$ admet une solution primitive.

Il a été aussi démontré dans le même article que $p(2)=2, p(3)=3$ et que pour tout $d \geq 4$ on a $p(d)>2$.

Cette étude va nous permettre de démontrer que 37 est un majorant de $p(4)$.

2. Polynômes à plusieurs variables sur un corps fini à valeurs une puissance $e$-ième. On va commencer par énoncer le théorème de Carlitz [2]. 
2.1. ThÉORÈme. Pour tout $m \geq 1$ et pour tout diviseur e de $m$, il existe un entier $\lambda(m, e)>m$ tel que si $q \geq \lambda(m, e)$ et e divise $q-1$, tout polynôme de $\mathbb{K}[X]$ de degré $m$ dont les valeurs sont des puissances e-ièmes dans $\mathbb{K}$ est une puissance e-ième d'un polynôme de $\mathbb{K}[X]$.

On généralise ce théorème au cas de polynômes à plusieurs variables comme suit :

2.2. ThÉORÈme. Soient $F \in \mathbb{K}\left[X_{1}, \ldots, X_{n}\right]$ de degré $m$ tel que $F(x)$ soit une puissance e-ième dans $\mathbb{K}$ pour tout $x \in \mathbb{K}^{n}$ et e un diviseur de $m$. Si $q \geq$ $\lambda(m, e)$ et e divise $q-1$, alors il existe $f \in \mathbb{K}\left[X_{1}, \ldots, X_{n}\right]$ vérifiant $F=f^{e}$.

Preuve. Les cas $F=0$ et $e=1$ sont triviaux. On suppose maintenant que $F \neq 0$ et $e>1$. $F$ peut s'écrire $F=F_{0}+F_{1}+\ldots+F_{m}$ où $F_{i}$ désigne la composante homogène de $F$ de degré $i$ et $F_{m} \neq 0$. Par hypothèse $m<q$, donc $\operatorname{deg}_{i} F_{m} \leq q-1$ pour tout $i=1, \ldots, n$. Il existe alors $a \in \mathbb{K}^{n}-$ $\{(0, \ldots, 0)\}$ tel que $F_{m}(a) \neq 0$.

Considérons une transformation linéaire, homogène et inversible des variables $X_{i}$ transformant $a$ en $(1,0, \ldots, 0)$. On peut écrire $X_{i}=\sum_{j=1}^{n} a_{i j} Y_{j}$. Posons $G_{m}(Y)=F_{m}(X)$. On a

$G_{m}(Y)$

$=\alpha Y_{1}^{m}+Y_{1}^{m-1} A_{1}\left(Y_{2}, \ldots, Y_{n}\right)+\ldots+Y_{1} A_{m-1}\left(Y_{2}, \ldots, Y_{n}\right)+A_{m}\left(Y_{2}, \ldots, Y_{n}\right)$ où $G_{m}((1,0, \ldots, 0)) \neq 0, A_{i}$ désigne une forme de degré $i$ et $\alpha \in \mathbb{K}^{*}$. En appliquant la même transformation à $F_{i}$ pour $i=1, \ldots, m-1$, on obtient un élément $G_{i}$ de $\mathbb{K}\left[Y_{1}, \ldots, Y_{n}\right]$ tel que $F_{i}(X)=G_{i}(Y)$. D'où

$$
F(X)=\sum_{i=0}^{m-1} G_{i}(Y)+G_{m}(Y) \quad \text { et } \quad \operatorname{deg}_{1}\left(\sum_{i=1}^{m-1} G_{i}(Y)\right) \leq m-1 .
$$

Posons $F(X)=G(Y)$. On obtient

$G(Y)$

$=\alpha Y_{1}^{m}+Y_{1}^{m-1} f_{1}\left(Y_{2}, \ldots, Y_{n}\right)+\ldots+Y_{1} f_{m-1}\left(Y_{2}, \ldots, Y_{n}\right)+f_{m}\left(Y_{2}, \ldots, Y_{n}\right)$

où $f_{i} \in \mathbb{K}\left[Y_{2}, \ldots, Y_{n}\right]$ est un polynôme de degré inférieur ou égal à $i$.

Soit $\left(c_{2}, \ldots, c_{n}\right) \in \mathbb{K}^{n-1}$. Alors $G\left(Y_{1}, c_{2}, \ldots, c_{n}\right)$ est un élément de $\mathbb{K}\left[Y_{1}\right]$ de degré $m$ et $G\left(c_{1}, c_{2}, \ldots, c_{n}\right)$ est une puissance $e$-ième dans $\mathbb{K}$ pour tout $c_{1} \in \mathbb{K}$.

Comme $q \geq \lambda(m, e)$, d'après le théorème 2.1, il existe $P_{c_{2}, \ldots, c_{n}} \in \mathbb{K}\left[Y_{1}\right]$ tel que $G\left(Y_{1}, c_{2}, \ldots, c_{n}\right)=\left(P_{c_{2}, \ldots, c_{n}}\left(Y_{1}\right)\right)^{e}$. Par ailleurs, on peut supposer dans l'expression de $G$ que $\alpha=1$. Donc $P_{c_{2}, \ldots, c_{n}}$ peut s'écrire sous la forme $P_{c_{2}, \ldots, c_{n}}$

$$
=Y_{1}^{g}+B_{1}\left(c_{2}, \ldots, c_{n}\right) Y_{1}^{g-1}+\ldots+B_{g-1}\left(c_{2}, \ldots, c_{n}\right) Y_{1}+B_{g}\left(c_{2}, \ldots, c_{n}\right)
$$

où $B_{1}, \ldots, B_{g}$ sont des applications de $\mathbb{K}^{n-1}$ dans $\mathbb{K}$ et $g=m / e$. 
Considérons maintenant les polynômes $P_{i}(i=1, \ldots, g)$ définis par

$$
P_{i}\left(Y_{2}, \ldots, Y_{n}\right)=\sum_{\left(y_{2}, \ldots, y_{n}\right) \in \mathbb{K}^{n-1}} B_{i}\left(y_{2}, \ldots, y_{n}\right) \prod_{j=2}^{n}\left(1-\left(Y_{j}-y_{j}\right)^{q-1}\right) .
$$

Montrons que

$G\left(Y_{1}, \ldots, Y_{n}\right)$

$=\left(Y_{1}^{g}+P_{1}\left(Y_{2}, \ldots, Y_{n}\right) Y_{1}^{g-1}+\ldots+P_{g-1}\left(Y_{2}, \ldots, Y_{n}\right) Y_{1}+P_{g}\left(Y_{2}, \ldots, Y_{n}\right)\right)^{e}$.

Pour cela on a besoin de la proposition suivante [4, p. 144] :

Proposition. Soit $f \in \mathbb{K}\left[X_{1}, \ldots, X_{n}\right]$. Il existe un polynôme unique $\bar{f} \in \mathbb{K}\left[X_{1}, \ldots, X_{n}\right]$ tel que $\operatorname{deg}_{i} \bar{f} \leq q-1$ pour tout $i=1, \ldots, n$ et $f \equiv$ $\bar{f}(\bmod I)$ où $I$ est l'idéal de $\mathbb{K}\left[X_{1}, \ldots, X_{n}\right]$ engendré par les polynômes $X_{1}^{q}-X_{1}, X_{2}^{q}-X_{2}, \ldots, X_{n}^{q}-X_{n}$.

Par construction $P_{i}$ est à la forme réduite et $P_{i}\left(c_{2}, \ldots, c_{n}\right)=B_{i}\left(c_{2}, \ldots, c_{n}\right)$ pour tout $\left(c_{2}, \ldots, c_{n}\right) \in \mathbb{K}^{n-1}$. Par suite, on a

$$
\begin{aligned}
G\left(Y_{1}, \ldots, Y_{n}\right) \equiv & \left(Y_{1}^{g}+P_{1}\left(Y_{2}, \ldots, Y_{n}\right) Y_{1}^{g-1}+\ldots\right. \\
& \left.\ldots+P_{g-1}\left(Y_{2}, \ldots, Y_{n}\right) Y_{1}+P_{g}\left(Y_{2}, \ldots, Y_{n}\right)\right)^{e}(\bmod I)
\end{aligned}
$$

où $I$ est l'idéal engendré par $Y_{1}^{q}-Y_{1}, \ldots, Y_{n}^{q}-Y_{n}$.

D'autre part, comme

$$
\begin{aligned}
\left(Y_{1}^{g}+P_{1}\left(Y_{2}, \ldots, Y_{n}\right) Y_{1}^{g-1}\right. & \left.+\ldots+P_{g-1}\left(Y_{2}, \ldots, Y_{n}\right) Y_{1}+P_{g}\left(Y_{2}, \ldots, Y_{n}\right)\right)^{e} \\
& =Y_{1}^{m}+\sum_{r=1}^{m}\left(\sum_{i_{1}+\ldots+i_{e}=r} P_{i_{1}} \ldots P_{i_{e}}\right) Y_{1}^{m-r}
\end{aligned}
$$

on a

$$
\begin{aligned}
Y_{1}^{m}+f_{1} Y_{1}^{m-1}+\ldots & +f_{m-1} Y_{1}+f_{m} \\
& \equiv Y_{1}^{m}+\sum_{r=1}^{m}\left(\sum_{i_{1}+\ldots+i_{e}=r} P_{i_{1}} \ldots P_{i_{e}}\right) Y_{1}^{m-r}(\bmod I) .
\end{aligned}
$$

Par conséquent,

$$
\sum_{r=1}^{m} \overline{\left(f_{r}-\sum_{i_{1}+\ldots+i_{e}=r} P_{i_{1}} \ldots P_{i_{e}}\right)} Y_{1}^{m-r} \equiv 0(\bmod I) .
$$

Or $\operatorname{deg}_{i} \sum_{r=1}^{m} \overline{\left(f_{r}-\sum_{i_{1}+\ldots+i_{e}=r} P_{i_{1}} \ldots P_{i_{e}}\right)} Y_{1}^{m-r} \leq q-1 \quad$ pour tout $i=1, \ldots, n$. 
D'où

$$
\sum_{r=1}^{m} \overline{\left(f_{r}-\sum_{i_{1}+\ldots+i_{e}=r} P_{i_{1}} \ldots P_{i_{e}}\right)} Y_{1}^{m-r}=0
$$

(d'après la proposition précédente), donc

$$
f_{r} \equiv \sum_{i_{1}+\ldots+i_{e}=r} P_{i_{1}} \ldots P_{i_{e}}(\bmod I) .
$$

Par récurrence sur $r$ on montre que

$$
f_{r}=\sum_{i_{1}+\ldots+i_{e}=r} P_{i_{1}} \ldots P_{i_{e}} \quad \text { et } \quad \operatorname{deg} P_{i_{j}} \leq i_{j} \quad \text { pour } i_{j} \leq r
$$

(pour tout $r=1, \ldots, m$ ).

Finalement,

$G\left(Y_{1}, \ldots, Y_{n}\right)$

$=\left(Y_{1}^{g}+P_{1}\left(Y_{2}, \ldots, Y_{n}\right) Y_{1}^{g-1}+\ldots+P_{g-1}\left(Y_{2}, \ldots, Y_{n}\right) Y_{1}+P_{g}\left(Y_{2}, \ldots, Y_{n}\right)\right)^{e}$.

En revenant aux variables $X_{1}, \ldots, X_{n}$, on obtient un polynôme $f \in$ $\mathbb{K}\left[X_{1}, \ldots, X_{n}\right]$ tel que $F=f^{e}$.

D'autre part, dans [1] Carlitz a démontré le résultat suivant:

Si f est un polynôme de $\mathbb{K}[X]$ de degré $m$ pair, si $q>(m-1)^{2}$ et si les valeurs prises par $f$ sur $\mathbb{K}$ sont toujours des carrés non nuls, alors $f$ est un carré dans $\mathbb{K}[X]$.

Dans ce qui suit, on se propose de généraliser ce résultat au cas où $f$ prend des valeurs carrées quelconques. Ceci va nous permettre de donner un majorant de $\lambda(m, 2)$.

2.3. ThÉORÈme. Soit $f \in \mathbb{K}[X]$ de degré $m$ tel que $m^{2}<q$. Si les valeurs prises par $f$ sur $\mathbb{K}$ sont toujours des carrés, alors $m$ est pair et il existe $g \in \mathbb{K}[X]$ tel que $f=g^{2}$.

Pr e u ve. Supposons qu'il n'existe pas $g \in \mathbb{K}[X]$ tel que $f=g^{2}$. D'après le théorème $2 \mathrm{c}^{\prime}$ de $[6, \mathrm{p} .43]$ on a

$$
\left|\sum_{x \in \mathbb{K}} \chi(f(x))\right| \leq(N-1) q^{1 / 2}
$$

où $\chi$ est un caractère d'ordre 2 de $\mathbb{K}^{*}$ et $N$ le nombre de racines distinctes de $f$ dans $\mathbb{K}$.

Or pour tout $x \in \mathbb{K}$, si $f(x)=0$, on a $\chi(f(x))=0$ et si $f(x) \neq 0$, on a $\chi(f(x))=1$ puisque $f(x)$ est un carré dans $\mathbb{K}$. D'où $\sum_{x \in \mathbb{K}} \chi(f(x))=$ $q-N$. Ceci entraîne que $q-N \leq(N-1) \sqrt{q}$ et donne, en élevant au carré, $q^{2}-\left(2 N+(N-1)^{2}\right) q+N^{2} \leq 0$. Ainsi $q \leq N^{2}$, ce qui démontre le théorème. 


\section{Polynômes singuliers sur un corps fini à 9 variables au moins}

DÉfinition. Soient $\mathbb{K}$ un corps et $F$ un élément de $\mathbb{K}\left[X_{1}, \ldots, X_{n}\right]$ non nul et sans terme constant. On dit que $F$ est singulier si pour tout $x=$ $\left(x_{1}, \ldots, x_{n}\right) \in \mathbb{K}^{n}-\{(0, \ldots, 0)\}$ tel que $F(x)=0$, on a $\frac{\partial F}{\partial X_{i}}(x)=0$ pour tout $1 \leq i \leq n$.

3.1. ThÉORÈme. Soient $n$ un entier $\geq 9$ et $F$ un élément de $\mathbb{K}\left[X_{1}, \ldots, X_{n}\right]$ de degré 4 , singulier et sans terme constant. Si $q>36$, il existe un élément $v$ non carré de $\mathbb{K}$, et deux éléments $g_{1}$ et $g_{2}$ de $\mathbb{K}\left[X_{1}, \ldots, X_{n}\right]$ de degré $\leq 2$ et sans terme constant tels que

$$
F=\varepsilon\left(g_{1}^{2}-v g_{2}^{2}\right) \quad \text { ò̀ } \varepsilon= \pm 1 .
$$

Pour démontrer ce théorème on a besoin des lemmes suivants où $F$ désigne le polynôme défini dans le théorème 3.1.

3.2. Lemme. Il existe une transformation linéaire homogène et inversible des variables $X_{i}=L_{i}\left(Y_{1}, \ldots, Y_{n}\right)$ telle que

$$
\begin{aligned}
F\left(X_{1}, \ldots, X_{n}\right) & =G\left(Y_{1}, \ldots, Y_{n}\right) \\
& =Y_{1}^{2} Q\left(Y_{2}, \ldots, Y_{n}\right)+2 Y_{1} C\left(Y_{2}, \ldots, Y_{n}\right)+U\left(Y_{2}, \ldots, Y_{n}\right)
\end{aligned}
$$

où $G, Q, C$ et $U$ sont des éléments de $\mathbb{K}\left[X_{1}, \ldots, X_{n}\right]$ vérifiant les propriétés suivantes: $G$ dépend de $Y_{1}, Q$ est une forme quadratique, $C$ et $U$ sont sans composante homogène de degré $\leq 1$ et respectivement de degré $\leq 3$ et $\leq 4$.

Pr e u v e. Comme $F$ est singulier, la composante homogène de degré 1 est nulle [3]. Ainsi $F=F_{4}+F_{3}+F_{2}$ où $F_{i}$ représente la composante homogène de degré $i$ de $F$.

D'après le théorème de Warning le nombre d'éléments dans $\mathbb{K}^{n}$ qui vérifient $F_{4}(x)=0$ et $\left(F_{3}+F_{2}\right)(x)=0$ est supérieur ou égal à $q^{n-7}$. Dès lors, il existe $x_{0}, x_{1}$ et $x_{2}$ éléments de $\mathbb{K}^{n}-\{(0, \ldots, 0)\}$ linéairement indépendants tels que $x_{0}$ et $x_{1}$ soient des zéros communs de $F_{4}$ et de $F_{3}+F_{2}$ et $F_{4}\left(x_{2}\right) \neq 0$. Il existe alors une transformation homogène linéaire et inversible des variables $X_{i}=u_{i}\left(Z_{1}, \ldots, Z_{n}\right)$ qui transforme $(1,1, \ldots, 0),(-1,1,0, \ldots, 0)$ et $(1,0, \ldots, 0)$ respectivement en $x_{0}, x_{1}$ et $x_{2}$.

Posons $H\left(Z_{1}, \ldots, Z_{n}\right)=F\left(X_{1}, \ldots, X_{n}\right)=F\left(u_{1}, \ldots, u_{n}\right)$. Alors $H$ peut s'écrire sous la forme

$$
H=a_{1} Z_{1}^{4}+a_{2} Z_{1}^{3} Z_{2}+a_{3} Z_{1}^{2} Z_{2}^{2}+a_{4} Z_{1} Z_{2}^{3}+a_{5} Z_{2}^{4}+R+H_{3}+H_{2}
$$

où $a_{1} \neq 0, R$ est une forme nulle ou de degré 4 n'admettant pas de monômes de la forme $\alpha Z_{1}^{n_{1}} Z_{2}^{n_{2}}$, et $H_{3}$ et $H_{2}$ sont les composantes homogènes de degré respectivement 3 et 2 .

Considérons $G_{1}$ et $G_{2}$ définis par $G_{1}\left(Y_{1}, \ldots, Y_{n}\right)=H\left(Y_{1}, Y_{1}+Y_{2}, Y_{3}, \ldots\right.$ $\left.\ldots, Y_{n}\right)$ et $G_{2}=H\left(Y_{1},-Y_{1}+Y_{2}, Y_{3}, \ldots, Y_{n}\right)$. Ils s'écrivent alors sous la forme 


$$
\begin{aligned}
G_{1}= & \left(a_{1}+a_{2}+a_{3}+a_{4}+a_{5}\right) Y_{1}^{4}+\left(a_{2}+2 a_{3}+3 a_{4}+4 a_{5}\right) Y_{1}^{3} Y_{2} \\
& +\left(a_{3}+3 a_{4}+6 a_{5}\right) Y_{1}^{2} Y_{2}^{2}+\left(a_{4}+4 a_{5}\right) Y_{1} Y_{2}^{3}+a_{5} Y_{2}^{4}+R^{\prime}+H_{2}^{\prime}+H_{3}^{\prime}, \\
G_{2}= & \left(a_{1}-a_{2}+a_{3}-a_{4}+a_{5}\right) Y_{1}^{4}+\left(a_{2}-2 a_{3}+3 a_{4}-4 a_{5}\right) Y_{1}^{3} Y_{2} \\
& +\left(a_{3}-3 a_{4}+6 a_{5}\right) Y_{1}^{2} Y_{2}^{2}+\left(a_{4}-4 a_{5}\right) Y_{1} Y_{2}^{3}+a_{5} Y_{2}^{4}+R^{\prime \prime}+H_{2}^{\prime \prime}+H_{3}^{\prime \prime}
\end{aligned}
$$

où $R^{\prime}$ et $R^{\prime \prime}$ sont des formes nulles ou de degré 4 et ne contiennent pas de monômes de la forme $\alpha Y_{1}^{n_{1}} Y_{2}^{n_{2}}$, et $H_{2}^{\prime}, H_{3}^{\prime}, H_{2}^{\prime \prime}$ et $H_{3}^{\prime \prime}$ sont des formes de degré $\leq 3$. Dès lors, $G_{1}$ ou $G_{2}$ va dépendre de $Y_{1}$ : sinon $a_{1}=a_{2}=a_{3}=$ $a_{4}=a_{5}=0$, ce qui contredit le fait que $a_{1} \neq 0$.

Soit $G$ le polynôme qui dépend de $Y_{1}$. $G$ peut s'écrire

$$
\begin{aligned}
G= & a Y_{1}^{4}+b Y_{1}^{3}+c Y_{1}^{2}+Y_{1}^{3}\left(\alpha_{2} Y_{2}+\ldots+\alpha_{n} Y_{n}\right) \\
& +Y_{1}^{2}\left(Q\left(Y_{2}, \ldots, Y_{n}\right)+\beta_{2} Y_{2}+\ldots+\beta_{n} Y_{n}\right) \\
& +Y_{1}\left(C\left(Y_{2}, \ldots, Y_{n}\right)+\gamma_{2} Y_{2}+\ldots+\gamma_{n} Y_{n}\right)+U\left(Y_{2}, \ldots, Y_{n}\right) .
\end{aligned}
$$

D'après ce qui précède, la composante homogène de degré 4 de $G$ vérifie $G_{4}(1,0, \ldots, 0)=0$, d'où $a=0$. D'autre part, on a les relations

$$
\begin{gathered}
G(1,0, \ldots, 0)=b+c=0, \\
\frac{\partial G}{\partial Y_{1}}(1,0, \ldots, 0)=3 b+2 c=0 .
\end{gathered}
$$

(2) est vérifiée puisque $G$ est singulier comme $F$. A partir de (1) et (2) nous déduisons que $b=c=0$.

Dès lors, $G(x, 0, \ldots, 0)=0$ pour tout $x \in \mathbb{K}$, et comme $G$ est singulier on a

$$
\frac{\partial G}{\partial Y_{i}}(x, 0, \ldots, 0)=\alpha_{i} x^{3}+\beta_{i} x^{2}+\gamma_{i} x=0 \quad \text { pour tout } i \in\{2, \ldots, n\} .
$$

Comme Card $\mathbb{K}>3$, on en déduit que $\alpha_{i}=\beta_{i}=\gamma_{i}=0$ pour tout $i \in$ $\{2, \ldots, n\}$, d'où le lemme.

3.3. Lemme. (i) Soit $y \in \mathbb{K}^{n-1}$. Si $Q(y)=0$, alors $C(y)=0$.

(ii) $Q$ est une forme quadratique non nulle.

(iii) $U$ est singulier.

(iv) Soit $y \in \mathbb{K}^{n-1}$. Si $U(y)=0$, alors $C(y)=0$.

Preuve. (i) Si $C(y) \neq 0$, on a

$$
G\left(\frac{-U(y)}{2 C(y)}, y\right)=0
$$

ce qui donne

$$
\frac{\partial G}{\partial Y_{1}}\left(\frac{-U(y)}{2 C(y)}, y\right)=\frac{-2 U(y)}{C(y)} Q(y)+2 C(y) \neq 0 .
$$

Ceci contredit le fait que $G$ est singulier. 
(ii) Si $Q$ est nulle, $C$ est nulle d'après (i). Ceci contredit que $G$ dépend de $Y_{1}$.

(iii) et (iv) sont des conséquences du fait que $G$ est singulier.

Dans la suite posons $\Delta=C^{2}-Q U$.

3.4. Lemme. Si $\Delta=v D^{2}$ où $D \in \mathbb{K}\left[Y_{2}, \ldots, Y_{n}\right]$ de degré $\leq 3$ et $v \in \mathbb{K}$, alors $Q$ est une forme singulière.

Preuve. Soit $y \in \mathbb{K}^{n-1}$ tel que $Q(y)=0$. En vertu du lemme 3.3, $C(y)=0$ et donc $D(y)=0$. Par conséquent, $U \frac{\partial Q}{\partial Y_{i}}(y)=0$.

- Si $U(y)=0$, alors $y$ est un zéro singulier de $Q$. En effet, supposons qu'il existe $i_{0} \in\{2, \ldots, n\}$ tel que $\frac{\partial Q}{\partial Y_{i_{0}}}(y) \neq 0$. Considérons $a \in \mathbb{K}$ tel que

$$
a^{2} \frac{\partial Q}{\partial Y_{i_{0}}}(y)+2 a \frac{\partial C}{\partial Y_{i_{0}}}(y) \neq 0 .
$$

On a alors $G(a, y)=0$ et $\frac{\partial G}{\partial Y_{i_{0}}}(a, y) \neq 0$, ce qui est absurde.

- Si $U(y) \neq 0$, alors $\frac{\partial Q}{\partial Y_{i}}(y)=0$.

3.5. Lemme. Il existe $\mu \in \mathbb{K}^{*}$ non carré tel que pour tout $y \in \mathbb{K}^{n-1}$, $\mu \Delta(y)$ soit un carré dans $\mathbb{K}$.

Preuve. Soit $\mu \in \mathbb{K}^{*}$ et non carré. S'il existe $y \in \mathbb{K}^{n-1}$ tel que $\mu \Delta(y)$ soit non carré dans $\mathbb{K}$, alors $\mu \Delta(y) \mu^{-1}=\Delta(y)$ est un carré non nul et il existe $k \in \mathbb{K}^{*}$ tel que $\Delta(y)=k^{2}$. Comme $\Delta(y) \neq 0$, alors $Q(y) \neq 0$. Dès lors, l'équation $Y_{1}^{2} Q(y)+2 Y_{1} C(y)+U(y)=0$ admet comme racine $(k-C(y)) / Q(y)$. Ainsi $((k-C(y)) / Q(y), y)$ serait un zéro non singulier de $G$, ce qui est absurde.

3.6. Lemme. Soit $Q \in \mathbb{K}\left[Y_{2}, \ldots, Y_{n}\right]$ une forme quadratique. Si $Q$ est une forme singulière, elle peut s'écrire sous la forme $Q(Y)=\varepsilon\left[L_{1}^{2}-v L_{2}^{2}\right]$ où $\varepsilon= \pm 1$ et $v$ est non carré dans $\mathbb{K}, L_{1}$ et $L_{2}$ étant deux formes linéaires de $\mathbb{K}\left[Y_{2}, \ldots, Y_{n}\right]$.

La preuve résulte du lemme 3.2 de [3].

Preuve du thé or ème 3.1. D'après le lemme 3.2, il existe une transformation linéaire homogène et inversible des variables telle que $F\left(X_{1}, \ldots\right.$ $\left.\ldots, X_{n}\right)=G\left(Y_{1}, \ldots, Y_{n}\right)$ où $G$ dépend de $Y_{1}$ et s'écrit

(3) $G\left(Y_{1}, \ldots, Y_{n}\right)=Y_{1}^{2} Q\left(Y_{2}, \ldots, Y_{n}\right)+2 Y_{1} C\left(Y_{2}, \ldots, Y_{n}\right)+U\left(Y_{2}, \ldots, Y_{n}\right)$.

$1 \mathrm{er}$ cas : Si $U=0$ alors, d'après le lemme $3.3, C(a)=0$ pour tout $a \in \mathbb{K}^{n-1}$. $C$ est alors nul puisque $\operatorname{deg} C \leq 3<$ Card $\mathbb{K}$. Par suite, $G(Y)=$ $Y_{1}^{2} Q\left(Y_{2}, \ldots, Y_{n}\right)$ et $Q$ est singulière. En vertu du lemme 3.6, $Q$ s'écrit $Q=$ $\varepsilon\left[L_{1}^{2}-v L_{2}^{2}\right]$ où $\varepsilon= \pm 1$ et $v$ est un élément non carré de $\mathbb{K}, L_{1}$ et $L_{2}$ étant deux formes linéaires de $\mathbb{K}\left[Y_{2}, \ldots, Y_{n}\right]$. Donc

$$
G=\varepsilon Y_{1}^{2}\left[L_{1}^{2}-v L_{2}^{2}\right]=\varepsilon\left[\left(Y_{1} L_{1}\right)^{2}-v\left(Y_{1} L_{2}\right)^{2}\right] .
$$


Par la transformation inverse, $F$ peut s'écrire sous la forme

$$
F(X)=\varepsilon\left[g_{1}(X)^{2}-v g_{2}(X)^{2}\right] .
$$

2 - ième cas : Si $U \neq 0$, d'après le lemme 3.3 on a $Q \neq 0$. Montrons que $\Delta=v D^{2}$ où $v \in \mathbb{K}$ est non carré et $D \in \mathbb{K}\left[Y_{2}, \ldots, Y_{n}\right]$ est de degré $\leq 3$ sans terme constant.

D'après le lemme 3.5 , il existe $\lambda \in \mathbb{K}$ non carré tel que $\lambda \Delta(a)$ soit un carré dans $\mathbb{K}$ pour tout $a \in \mathbb{K}^{n-1}$. De plus, si $d$ est le degré de $\lambda \Delta$, on a $d \leq 6<\lambda(d, 2)<36 \leq$ Card $\mathbb{K}$. D'après le théorème 2.2, il existe alors $D \in \mathbb{K}\left[Y_{2}, \ldots, Y_{n}\right]$ de degré $\leq 3$ sans terme constant tel que $\lambda \Delta=D^{2}$, d'où le résultat en posant $v=1 / \lambda$. En vertu du lemme 3.4, $Q$ est singulière; elle peut s'écrire alors

$$
Q=\varepsilon\left[L_{1}^{2}-\mu L_{2}^{2}\right]
$$

où $\mu$ est non carré dans $\mathbb{K}$ et $\varepsilon= \pm 1$. Dès lors,

$$
Q=\varepsilon\left[L_{1}^{2}-v \mu v^{-1} L_{2}^{2}\right] .
$$

Comme $\mu v^{-1}$ est un carré dans $\mathbb{K}$, il existe une forme linéaire $L_{3}$ vérifiant $\mu v^{-1} L_{2}^{2}=L_{3}^{2}$. Par suite, $Q=\varepsilon\left[L_{1}^{2}-v L_{3}^{2}\right]$.

Montrons que

$$
C=L_{1} q_{1}-v L_{3} q_{2}
$$

où $q_{1}$ et $q_{2}$ sont deux éléments de $\mathbb{K}\left[Y_{2}, \ldots, Y_{n}\right]$ de degré $\leq 2$ et sans terme constant.

En décomposant $Q$ et $C^{2}-v D^{2}$ dans $\mathbb{K}\left(v^{1 / 2}\right)\left[Y_{2}, \ldots, Y_{n}\right]$ on a

$$
\varepsilon\left(L_{1}-v^{1 / 2} L_{3}\right)\left(L_{1}+v^{1 / 2} L_{3}\right) U=\left(C-v^{1 / 2} D\right)\left(C+v^{1 / 2} D\right) .
$$

Donc $L_{1}-v^{1 / 2} L_{3}$ divise $\left(C-v^{1 / 2} D\right)\left(C+v^{1 / 2} D\right)$. On a aussi

$$
C-v^{1 / 2} D \neq 0 \quad \text { et } \quad C+v^{1 / 2} D \neq 0 .
$$

En effet, si $C+v^{1 / 2} D=0$ ou $C-v^{1 / 2} D=0$, alors $C=0$ et $D=0$ puisque $\left(1, v^{1 / 2}\right)$ est une base de $\mathbb{K}\left[v^{1 / 2}\right]$ considéré comme $\mathbb{K}$-espace vectoriel. On a alors $Q U=C^{2}-v D^{2}=0$ et par conséquent $Q=0$, ce qui est impossible (lemme 3.3).

En choisissant donc d'avance un signe de $D$, c'est-à-dire $\pm D$, on peut supposer que $L_{1}-v^{1 / 2} L_{3}$ divise $C+v^{1 / 2} D$. Il existe alors $q^{\prime} \in \mathbb{K}\left[v^{1 / 2}\right]\left[Y_{2}, \ldots, Y_{n}\right]$ de degré $\leq 2$ tel que $C+v^{1 / 2} D=\left(L_{1}-v^{1 / 2} L_{3}\right) q^{\prime}$. Par ailleurs, $q^{\prime}$ peut s'écrire $q^{\prime}=q_{1}+v^{1 / 2} q_{2}$ où $q_{1}$ et $q_{2}$ sont des éléments de $\mathbb{K}\left[Y_{2}, \ldots, Y_{n}\right]$ de degré $\leq 2$ et sans terme constant. Ainsi

$$
C+v^{1 / 2} D=\left(L_{1}-v^{1 / 2} L_{3}\right)\left(q_{1}+v^{1 / 2} q_{2}\right)
$$

et

$$
\left(C-L_{1} q_{1}+v L_{3} q_{2}\right)+\left(D-L_{1} q_{2}+L_{3} q_{1}\right) v^{1 / 2}=0 .
$$


Ceci entraîne que $\left(C-L_{1} q_{1}+v L_{3} q_{2}\right)(a)=0$ et $\left(D-L_{1} q_{2}+L_{3} q_{1}\right)(a)=0$ pour tout $a \in \mathbb{K}^{n-1}$.

D'autre part, on a $\operatorname{deg}\left(C-L_{1} q_{1}+v L_{3} q_{2}\right) \leq 3<\operatorname{Card} \mathbb{K}$, d'où $C-L_{1} q_{1}+$ $v L_{3} q_{2}=0$ et $C=L_{1} q_{1}-v L_{3} q_{2}$.

Montrons que $U=\varepsilon\left(q_{1}^{2}-v q_{2}^{2}\right)$. On a

$$
C+v^{1 / 2} D=\left(L_{1}-v^{1 / 2} L_{3}\right)\left(q_{1}+v^{1 / 2} q_{2}\right) .
$$

En appliquant à l'égalité (4) le $\mathbb{K}$-isomorphisme qui transforme $v^{1 / 2}$ en $-v^{1 / 2}$, on obtient

$$
C-v^{1 / 2} D=\left(L_{1}+v^{1 / 2} L_{3}\right)\left(q_{1}-v^{1 / 2} q_{2}\right) .
$$

Multiplions (4) et (5) membre à membre; on obtient

$$
C^{2}-v D^{2}=\varepsilon\left(L_{1}^{2}-v L_{3}^{2}\right)\left(q_{1}^{2}-v q_{2}^{2}\right) .
$$

Comme $Q=\varepsilon\left(L_{1}^{2}-v L_{3}^{2}\right), C^{2}-v D^{2}=\varepsilon Q\left(q_{1}^{2}-v q_{2}^{2}\right)$, on peut écrire

$$
Q U=C^{2}-v D^{2}=\varepsilon Q\left(q_{1}^{2}-v q_{2}^{2}\right)
$$

et par suite

$$
U=\varepsilon\left(q_{1}^{2}-v q_{2}^{2}\right) .
$$

Dans l'expression de $G$ donnée par (3), en remplaçant $Q, C$ et $U$ respectivement par $\varepsilon\left(L_{1}^{2}-v L_{3}^{2}\right), L_{1} q_{1}-v L_{3} q_{2}$ et $\varepsilon\left(q_{1}^{2}-v q_{2}^{2}\right)$, on obtient

$$
\begin{aligned}
G(Y) & =\varepsilon Y_{1}^{2}\left(L_{1}^{2}-v L_{3}^{2}\right)+2 Y_{1}\left(L_{1} q_{1}-v L_{3} q_{2}\right)+\varepsilon\left(q_{1}^{2}-v q_{2}^{2}\right) \\
& =\varepsilon\left[\left[Y_{1} L_{1}+\varepsilon q_{1}\right]^{2}-v\left[Y_{1} L_{3}+\varepsilon q_{2}\right]^{2}\right] .
\end{aligned}
$$

Finalement, par la transformation inverse, on a

$$
F=\varepsilon\left[g_{1}^{2}-v g_{2}^{2}\right]
$$

où $g_{1}$ et $g_{2}$ sont deux éléments de $\mathbb{K}\left[X_{1}, \ldots, X_{n}\right]$ de degré $\leq 2$ et sans terme constant.

\section{Les équations diophantiennes modulo $p^{2}$ de degré 4}

Définition. On dit que $\left(x_{1}, \ldots, x_{n}\right) \in \mathbb{Z}_{p}^{n}$ est primitif si l'un des $x_{i}$ n'est pas divisible par $p$.

4.1. ThÉORÈmE. Soit $p$ un nombre premier supérieur ou égal à 37. Alors pour tout $f \in \mathbb{Z}_{p}\left[X_{1}, \ldots, X_{9}\right]$ de degré quatre et sans terme constant, l'équation $f\left(x_{1}, \ldots, x_{9}\right) \equiv 0\left(\bmod p^{2}\right)$ admet une solution primitive.

Preuve. Considérons $F=\bar{f} \in \mathbb{F}_{p}\left[X_{1}, \ldots, X_{9}\right]$.

1 er cas : Si $F$ est nul, il existe $h \in \mathbb{Z}_{p}\left[X_{1}, \ldots, X_{9}\right]$ tel que $f=p h$. D'après le théorème de Chevalley-Warning, $h$ admet une solution primitive $\left(x_{1}, \ldots, x_{9}\right)$ qui vérifie $f\left(x_{1}, \ldots, x_{9}\right) \equiv 0\left(\bmod p^{2}\right)$. 
2 - i ème c a s : Si $F$ est non singulier, ils existent $\left(x_{1}, \ldots, x_{9}\right) \in \mathbb{F}_{p}^{9}$ et $1 \leq i_{0} \leq 9$ tels que

$$
F\left(x_{1}, \ldots, x_{9}\right)=0 \quad \text { et } \quad \frac{\partial F}{\partial X_{i_{0}}}\left(x_{1}, \ldots, x_{9}\right) \neq 0 .
$$

D'après le lemme de Hensel, il existe $\left(y_{1}, \ldots, y_{9}\right) \in \mathbb{Z}_{p}^{9}$ primitif tel que $f\left(y_{1}, \ldots, y_{9}\right)$ soit nul, ce qui implique $f\left(y_{1}, \ldots, y_{9}\right) \equiv 0\left(\bmod p^{2}\right)$.

3 - ième cas : Si $F$ est singulier et de degré 4 , écrivons $F=F_{4}+F_{3}+F_{2}$, où $F_{i}$ désigne la composante homogène de $F$ de degré $i$. D'après le théorème précédent, on a $F=\varepsilon\left[g_{1}^{2}-v g_{2}^{2}\right]$ où $g_{1}, g_{2} \in \mathbb{F}_{p}\left[X_{1}, \ldots, X_{9}\right]$ sont de degré $\leq 2$ et sans terme constant.

D'autre part, soient $g_{1}^{\prime}, g_{2}^{\prime} \in \mathbb{Z}_{p}\left[X_{1}, \ldots, X_{9}\right]$ de degré $\leq 2$ et sans terme constant tels que $\bar{g}_{1}^{\prime}=g_{1}$ et $\bar{g}_{2}^{\prime}=g_{2}$. Considérons $G \in \mathbb{Z}_{p}\left[X_{1}, \ldots, X_{9}\right]$ tel que

$$
f=\varepsilon\left(\left(g_{1}^{\prime}(X)\right)^{2}-v\left(g_{2}^{\prime}(X)\right)^{2}\right)+p G .
$$

Le système

$$
\begin{aligned}
& g_{1}^{\prime}\left(x_{1}, \ldots, x_{9}\right) \equiv 0(\bmod p), \\
& g_{2}^{\prime}\left(x_{1}, \ldots, x_{9}\right) \equiv 0(\bmod p), \\
& G\left(x_{1}, \ldots, x_{9}\right) \equiv 0(\bmod p)
\end{aligned}
$$

vérifie les hypothèses du théorème de Chevalley-Warning, il admet donc une solution primitive $\left(x_{1}, \ldots, x_{9}\right) \in \mathbb{Z}_{p}^{9}$. Cette solution vérifie $f\left(x_{1}, \ldots, x_{9}\right) \equiv$ $0\left(\bmod p^{2}\right)$.

4 - i ème c a s : Si $F$ est singulier et de degré $\leq 3$, les lemmes 3.2 et 4.1 de [3] permettent de démontrer le théorème dans ce cas.

Ainsi $p(4) \leq 37$; mais est-ce que 37 est une valeur optimale?

\section{Références}

[1] L. Carlitz, A problem of Dickson's, Duke Math. J. 14 (1947), 1139-1140.

[2] -, A problem of Dickson, ibid. 19 (1952), 471-474.

[3] E. M. Hanine, Équations diophantiennes modulo $p^{2}$, Colloq. Math. 64 (1993), $275-286$.

[4] K. Ireland and M. Rosen, Classical Introduction to Number Theory, 2nd ed., Springer, 1990.

[5] D. J. Lewis, Singular quartic forms, Duke Math. J. 21 (1954), 39-44.

[6] W. M. Schmidt, Equations over Finite Fields, An Elementary Approach, Lecture Notes in Math. 536, Springer, 1976.

LABORATOIRE D'ALGÈBRE

UNIVERSITÉ PAUL SABATIER

118 ROUTE DE NARBONNE

31062 TOULOUSE CEDEX, FRANCE 